\title{
Eléctrodos Modificados por Monocamadas Auto-Montadas
}

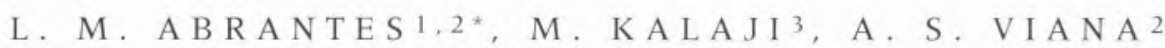

\begin{abstract}
O método de Auto-Montagem tem se mostrado muito eficaz na preparação de monocamadas em superficies sólidas, constituindo uma alternativa aos filmes moleculares de LangmuirBlodgett. A sua vantagem, para além da fácil utilização, é formar monocamadas estáveis,

organizadas e muito versáteis, logo apropriadas para diversas aplicações, tais como sensores, dispositivos electrónicos e em biotecnologia. Estes sistemas constituem modelos ideais para estudos de transferência electrónica em interfaces. A elevada estabilidade das monocamadas permite a sua caracterização por recurso a diversas técnicas electroquímicas e estruturais, in situ e ex situ.
\end{abstract}

\section{INTRODUÇÃO}

A formação de-superfícies modificadas por uma única camada de moléculas que partilhem de uma orientação comum e de elevado empacotamento, pode ser conseguida através de dois métodos: LangmuirBlodgett (LB) e Auto-Montagem ("Self-Assembly", SA).

O primeiro método a ser desenvolvido foi o LB, consistindo na construção de monocamadas através do contacto do substrato a modificar com uma camada previamente orientada, constituída por grupos hidrofílicos (cabeça) e hidrofóbicos (cauda), por compressão na interface ar-água [1-4].

As monocamadas auto-montadas ("Self-Assembled Monolayers", SAMs) são preparadas por adsorção espontânea de moléculas orgânicas num substrato, a partir de uma solução homogénea. A elevada organização da monocamada formada é conseguida pela afinidade existente entre um grupo (cabeça) e o substrato que estabelecem entre si uma ligação química, aliada à interacção favorável entre os grupos de cauda. De um modo geral, estes últimos são cadeias de alquilo, de comprimento va- riável, que mantêm entre si interacções do tipo Van der Waals $[5,6]$.

O método SA tem sido largamente estudado nas últimas duas décadas, adquirindo igual ou maior popularidade do que o LB, pois oferece certas vantagens, nomeadamente facilidade na preparação e elevada estabilidade das monocamadas. A grande versatilidade das monocamadas é obtida através da introdução de diferentes grupos funcionais, permitindo controlar certas propriedades, e.g. carácter hidrofóbico, topografia e propriedades redox [4]. Esta flexibilidade permite obter superfícies com as características necessárias a uma dada aplicação, que se mostram muito promissoras nas áreas de electrónica, construção de sensores, protecção à corrosão, lubrificação e biotecnologia, para além de serem modelos ideais para estudos mais fundamentais, como transferência electrónica em interfaces e cinéticas de adsorção $[5,7,8]$.

Nas monocamadas auto-montadas a interacção das moléculas com o substrato é frequentemente química, enquanto que no método LB a adsorção é de natureza física [5].

O primeiro sistema de SAMs a ser estudado foi a deposição de al-

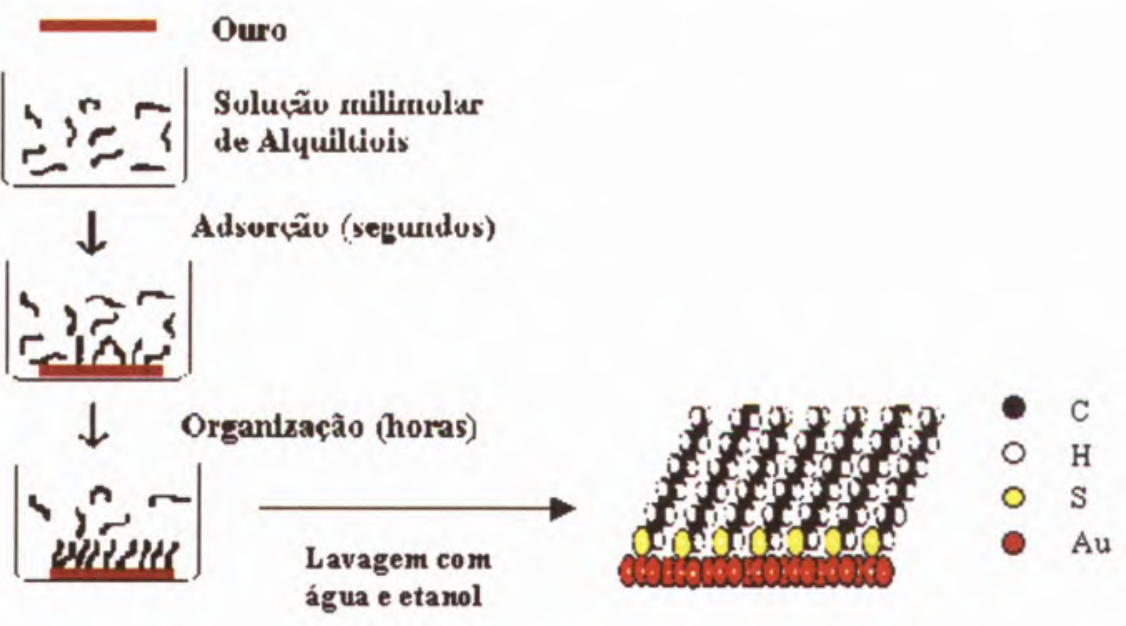

Fig. 1 - Esquema ilustrativo do procedimento utilizado na preparação das monocamadas auto-montadas. quilsilanos em vidro e óxido de alumínio, onde o grupo tricloro- ou trialquilsilano forma uma ligação covalente com os grupos $\mathrm{OH}$ do substrato [5]. Outros exemplos são ácidos gordos em óxidos metálicos, isocianetos em platina, e tióis, dissulfuretos e sulfuretos em ouro e platina [4].

Em particular, a interacção ouro-tiol conduz à formação termodinamicamente favorável da ligação ouro(I)-tiolato com a perda do hidrogénio, que se apresenta muito estável numa vasta gama de potencial. Esta característica torna esta classe ideal para o estudo electroquímico das SAMs [4,5,9].

\section{PREPARAÇÃO DAS MONOCAMADAS AUTO-MONTADAS}

A Figura 1 ilustra, de modo muito simplificado, o procedimento utilizado na preparação das SAMs, recorrendo ao sistema Au-Alquiltiol.

A qualidade da monocamada resultante, em termos de cobertura da superfície, organização e estabilidade, está relacionada com a morfologia e limpeza do substrato, e com as condições da deposição, concen- 
tração da solução, tempo de deposição e natureza do solvente $[5,7,10,11]$.

As soluções têm concentrações milimolares (1-50 mM) e o solvente mais usado é o etanol. Devido à forte interacção entre o ouro e o tiol, a adsorção da monocamada no substrato processa-se em alguns segundos. No entanto, são necessários tempos de adsorção de pelo menos 15 horas, para se obter uma monocamada estável e organizada. No esquema ilustrativo, "Adsorção (segundos)" corresponde à formação de uma monocamada incompleta e desorganizada, enquanto que "Organização (horas)" ilustra o estado de equilíbrio de adsorção de uma monocamada densa e organizada.

\section{CARACTERIZAÇÃO DAS MONOCAMADAS AUTO-MONTADAS}

A caracterização de monocamadas auto-montadas tem sido efectuada recorrendo a diversas técnicas electroquímicas, não electroquímicas in situ e ex situ. As mais utilizadas são Microbalança de Cristal de Quartzo (EQCM) [1,7,12-14], Espectroscopia de Infravermelho (IR) [6,15-17], Elipsometria [3,17-19], Microscopia de Túnel de Varrimento (STM) [9], Microscopia de Força Atómica (AFM) [20], Ângulo de Contacto (WCA) [3] e Espectroscopia Electrónica de Raios-X (XPS) [3,21].

Os diversos estudos de SAMs, por todas as técnicas mencionadas, indicam que os tiois com comprimentos de cadeias alquilo longas dão origem a monocamadas mais organizadas, compactas e estáveis do que compostos de cadeias menores. Quanto maior é a cadeia de hidrocarbonetos, mais elevado é o caracter hidrofóbico, mais fortes são as interacções entre os grupos de cauda e maior é a quantidade de espécies adsorvidas no substrato. Por este motivo, a grande maioria dos artigos publicados sobre estes sistemas incluem estudos com compostos de cadeias de alquilo superiores a 10 átomos de carbono $[5,8,13,17]$.
Os resultados obtidos por recurso a WCA, FTIR, STM e AFM, permitem elevado consenso no que respeita ao mecanismo de adsorção dos alquiltiois ao ouro, admitindo-se a perda do protão do tiol e a formação de uma sobrecamada com estrutura hexagonal $(\sqrt{3} \cdot \sqrt{3})$ R30 $[5,9]$. As cadeias apresentam uma configuração trans, com uma inclinação de $30^{\circ}$ com a normal à superfície, sendo a separação entre átomos de enxofre adjacentes de cerca de $5 \AA[5,22]$.

A Elipsometria Óptica tem sido utilizada para a determinação da espessura de monocamadas de alquiltiois $[3,17,18]$ e de alquiltiois substituídos [19,22]. A espessura da monocamada aumenta linearmente com o número de grupos metileno, $\mathrm{CH}_{2}$, (n), e a representação gráfica da espessura em função de $n$, indica que cada grupo $\mathrm{CH}_{2}$ tem $1.07 \AA ̊$ de comprimento $[3,17]$. A maioria dos estudos de elipsometria com monocamadas são efectuados ex-situ; no entanto. foram já realizadas experiências in situ onde a caracterização óptica está acoplada à electroquímica [19].

\section{COMPORTAMENTO ELECTROQUÍMICO}

As monocamadas auto-montadas de tiois em ouro podem ser classificadas, quanto ao seu comportamento electroquímico, em monocamadas electro-inactivas e electro-activas. No primeiro grupo, as monocamadas são constituídas por cadeias de hidrocarbonetos ligadas ao tiol e formam estruturas compactas capazes de bloquear a transferência electrónica entre o ouro e uma espécie electro-activa presente em solução [22].

As monocamadas electro-activas resultam da introdução de um grupo electro-activo, ligado covalentemente à cadeia de alquilo no extremo oposto ao tiol, dissulfureto ou sulfureto. As vantagens resultantes da presença destes grupos redox são várias, relevando a diminuição dos problemas associados à existência de poros $^{1}$ e defeitos 2 , quando se pretende estudar o efeito de túnel nos processos de transferência de electrónica. O empacotamento das moléculas à superfície determina a sua orientação, impedindo que os centros redox se movimentem livremente em direcção a um defeito ou poro $[5,22]$. Outra vantagem decorrente da utilização de centros redox, é a fácil determinação da quantidade de espécies adsorvidas no substrato, através de técnicas electroquímicas de uso corrente, aliada ao facto do estudo dos parâmetros electroquímicos de um sistema reversível fornecer informação sobre o ambiente que o circunda [8].

O modo como ocorre a transferência electrónica entre os centros redox adsorvidos e o eléctrodo tem sido largamente estudado [15,16,22-25], existindo várias propostas de mecanismos:

(i) Difusão do centro activo para um poro ou defeito, o que implica um movimento da molécula em direcção ao eléctrodo.

(ii) Os electrōes são trocados entre os centros activos, até serem conduzidos a poros ou defeitos.

(iii) Os electrões são transferidos por efeito de túnel ao longo das cadeias de hidrocarbonetos, até à superfície do eléctrodo.

Os estudos de SAMs in situ, por técnicas como Elipsometria, STM, e Espectroscopia de IV acopladas à electroquímica, são fundamentais para o conhecimento do mecanismo da transferência electrónica nestes sistemas, pois fornecem informação sobre a possível ocorrência de alterações na estrutura e orientação da monocamada, à medida que se faz variar o potencial do eléctrodo modificado.

No domínio das monocamadas electro-activas, um dos sistemas mais investigados envolve a adsorção de ferroceniltiois em ouro. A sua preferência deve-se ao facto do ferroceno (FC) se oxidar, num processo "reversível" e bem estudado a ferricínio $\left(\mathrm{FC}^{+}\right)[2,8]$. Os quatro grupos de ferroceniltiois (ou dissulfuretos) mais estudados (Figura 


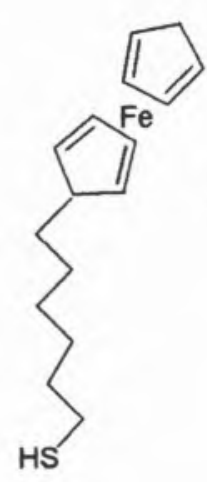

a)

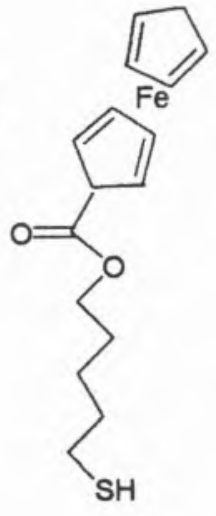

b)

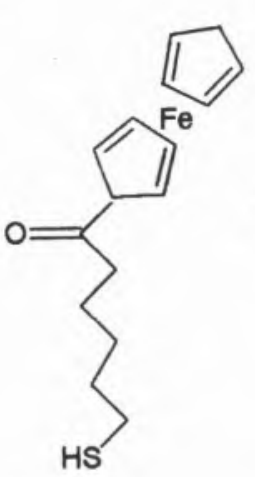

c)

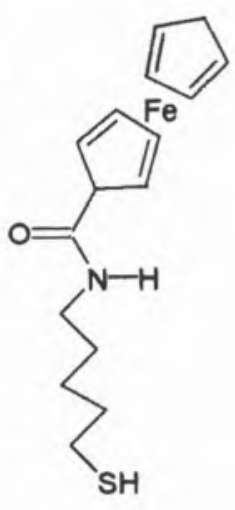

d)

Fig. 2 - Esquemas das estruturas moleculares dos compostos $\mathrm{Fc}\left(\mathrm{CH}_{2}\right)_{6} \mathrm{SH}$ (a), $\mathrm{FcCOO}\left(\mathrm{CH}_{2}\right)_{5} \mathrm{SH}\left(\right.$ b), $\mathrm{FcCO}\left(\mathrm{CH}_{2}\right)_{5} \mathrm{SH}$ (c) e $\mathrm{FcCONH}\left(\mathrm{CH}_{2}\right)_{5} \mathrm{SH}$ (d).

2) consistem na ligação da cadeia alquílica aos seguintes grupos: Fc (a), ao éster de Fc (FcCOO) (b), ao carbonilo de Fc (FcCO) (c) e ao grupo amida de Fc (FcCONH) (d) [5].

A natureza do grupo substituinte, juntamente com o comprimento da cadeia alquilo constituem parâmetros decisivos nas propriedades da monocamada a formar $[5,26]$. Hussey et al. [6] estudaram o comportamento electroquímico de ferroceniltiois e dissulfuretos com um grupo amida intercalado na cadeia alquilo. A presença do grupo amida possibilita a formação de pontes de hidrogénio entre os grupos alquilo, proporcionando uma maior estabilização e organização de monocamadas de cadeias mais curtas.

A voltametria cíclica tem sido a técnica electroquímica mais utilizada na caracterização de SAMs. A Figura 3 mostra os voltamogramas cíclicos e respectivas estruturas moleculares, obtidos para ferroceniltiois adsorvidos em ouro policristalino, com o substituinte carbonilo e uma cadeia de 3 (a), 5 (b) e 7 (c) átomos de carbono [27]. Por observação dos voltamogramas verifica-se que o tamanho da cadeia alquilo é determinante na forma dos picos redox do ferroceno.

De um modo geral, os parâmetros electroquímicos que se obtêm da análise dos voltamogramas deste tipo de sistemas são [5,6]:

(i) cobertura da superfície $\left(\Gamma_{\mathrm{Fc}}\right)$. A quantidade de composto adsorvido pode ser determinada pela integração da área dos picos, anódicos ou catódicos, dos processos redox. Os valores obtidos para os compostos (a),(b) e (c) foram $8.6 .10^{-11}, 3.2 .10^{-10}$ e $5.7 .10^{-10}$ molcm-2 ${ }^{-2}$ respectivamente [27]. É nítida a influência do tamanho da cadeia de alquilo na cobertura do eléctrodo.

(ii) potencial formal $\left(\mathrm{E}^{\mathrm{o}^{\prime}}\right)$. Este parâmetro reflecte a maior ou menor dificuldade que o centro redox tem para se oxidar e reduzir à superfície do eléctrodo modificado. Os valores de $E^{0^{\prime}}$ obtidos para os compostos (a) (b) e (c) à velocidade de varrimento de potencial de $100 \mathrm{mVs}^{-1}$ foram respectivamente $565( \pm 25 \mathrm{mV}), 570$ e $580 \mathrm{mV}$ $( \pm 5 \mathrm{mV})$ vs ESC, $0.1 \mathrm{M} \mathrm{HClO}_{4}$ [27]. Apesar das diferenças observadas não serem significativas, podem estar relacionadas com o facto de a monocamada formada por (a) ser menos organizada e menos compacta, permitindo um movimento mais livre dos grupos redox e uma maior penetração do electrólito suporte na monocamada. A introdução de um grupo doador de electrões, como é o caso do grupo carbonilo, vai desviar os potenciais formais para valores mais positivos do que compostos homólogos sem a presença de grupos substituintes. Uosaki et al [11] verifi- caram que nas mesmas condições os compostos $\mathrm{FCCO}\left(\mathrm{CH}_{2}\right)_{3} \mathrm{SH}$ e $\mathrm{Fc}\left(\mathrm{CH}_{2}\right)_{4} \mathrm{SH}$ quando adsorvidos no ouro, apresentavam valores de $\mathrm{E}^{\mathrm{o}^{\prime}}$ de 525 e $210 \mathrm{mV}$ (vs ESC; $1 \mathrm{M} \mathrm{HClO}_{4}$ ), respectivamente.

(iii) largura do pico anódico a meia altura do máximo da corrente $\left(\Delta \mathbf{E}_{\mathrm{FWMH}}\right)$. Este parâmetro fornece informação sobre as interacções existentes entre as moléculas electroactivas na monocamada. O valor esperado para $\Delta \mathrm{E}_{\mathrm{FWMH}}$, num caso ideal onde as interacções entre os centros redox são mínimas, é $3.53 \mathrm{RT} / \mathrm{nF}$ (90.3 mV a $\left.24^{\circ} \mathrm{C}[6]\right)$. Os valores experimentais retirados dos voltamogramas da Figura 3, foram 115 e $100 \mathrm{mV}$ para os compostos (b) e (c), respectivamente [27]. A onda anódica obtida para a oxidação do ferroceno na monocamada formada pelo composto (a) não apresenta um pico bem definido, e como tal não foi possível retirar um valor preciso de $\left(\Delta \mathrm{E}_{\mathrm{FWMH}}\right)$. O facto dos valores obtidos serem ligeiramente mais elevados do que o esperado, poderá ser atribuído à existência de vários potenciais formais com valores muito próximos; ou seja, à existência de centros redox fora e dentro da monocamada. No entanto, observa-se que o desvio é pequeno, indicando que a monocamada está bastante organizada.

(iv) separação entre os picos anódico e catódico $\left(\Delta \mathrm{E}_{\mathrm{p}}\right)$. É possivel avaliar qualitativamente a velocidade a que ocorre a transferência electrónica entre os centros redox e o eléctrodo modificado através do valor de $\Delta \mathrm{E}_{\mathrm{p}}$. O valor de $\Delta \mathrm{E}_{\mathrm{p}}$ esperado para uma espécie adsorvida à superfície do eléctrodo. com um comportamento reversível, deverá ser de $0 \mathrm{~V}$. Os valores de $\Delta \mathrm{E}_{\mathrm{p}}$ medidos para os compostos (b) e (c) foram 40 e $0 \mathrm{mV}( \pm 5 \mathrm{mV})$, respectivamente [27]. Os baixos valores observados comprovam que as espécies estão adsorvidas à superfície do eléctrodo e que a transferência electrónica é rápida. $\mathrm{O}$ valor de $\Delta \mathrm{E}_{\mathrm{p}}$ obtido para o composto (a) foi cerca de $80 \mathrm{mV}$ $( \pm 25 \mathrm{mV})$. Este valor, superior ao esperado para espécies adsorvidas, é 

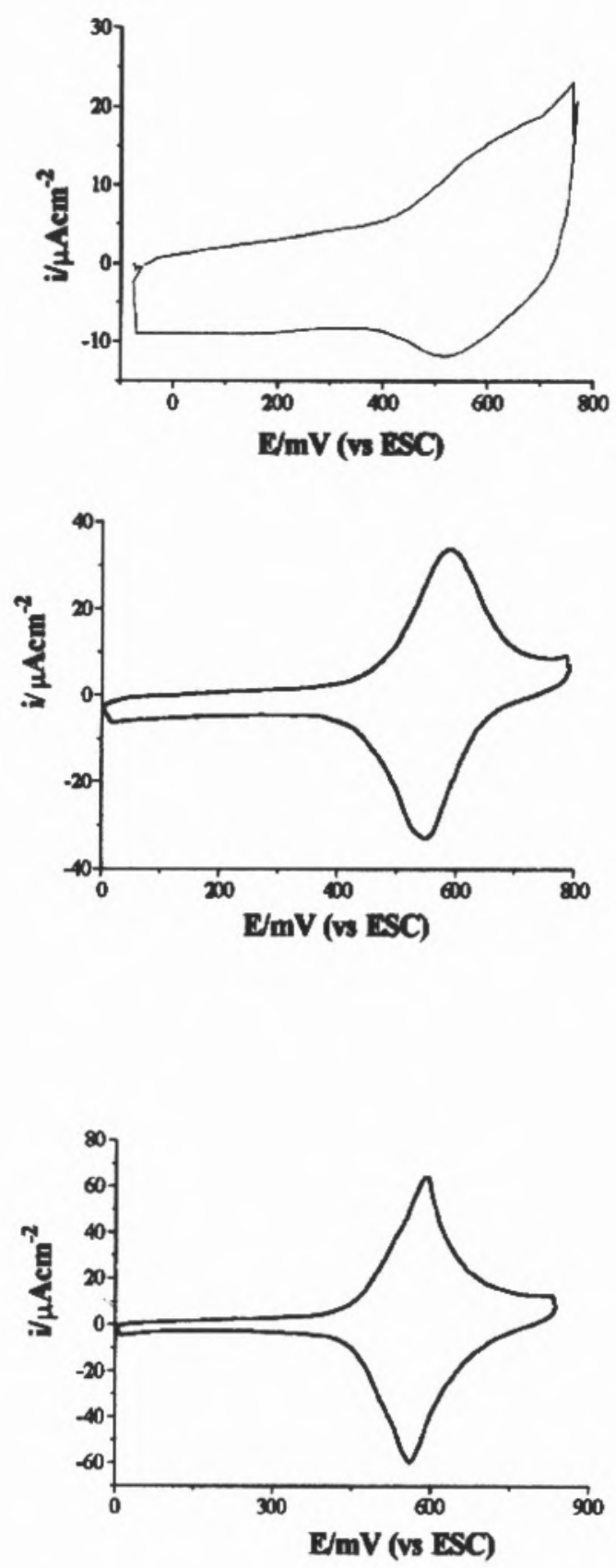

Fig. 3 - Voltamogramas cíclicos e representações moleculares $(\mathrm{C} \bullet, \mathrm{Fe} \bullet, \mathrm{H} \bullet, \mathrm{O} \bullet$, and $\mathrm{S} \bullet$ ), obtidos para monocamadas dos compostos $\mathrm{FcCO}\left(\mathrm{CH}_{2}\right)_{3} \mathrm{SH}(\mathrm{a}), \mathrm{FcCO}\left(\mathrm{CH}_{2}\right)_{5} \mathrm{SH}$ (b) e FcCO $\left(\mathrm{CH}_{2}\right)_{7} \mathrm{SH}$ (c) em ouro policristalino, em $0.1 \mathrm{M} \mathrm{HClO}_{4}$. Os voltamogramas foram registados a 100 $\mathrm{mVs}^{-1}$ [27].

apenas uma aproximação visto os picos redox do ferroceno estarem mal definidos no voltamograma do composto (a).

(v) Variação da intensidade
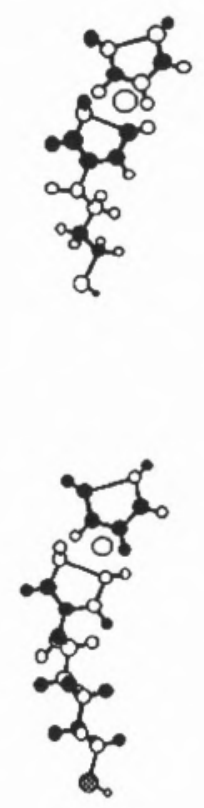

a)

b)

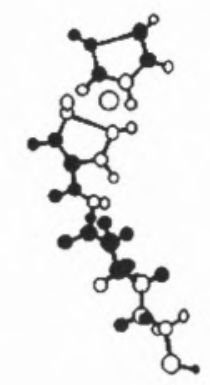

c)

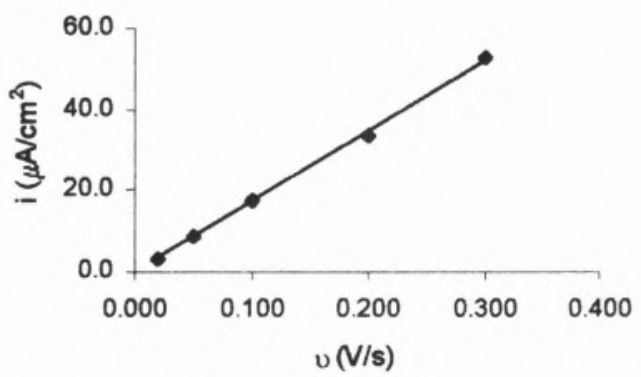

Fig. 4 - Representação gráfica da densidade de corrente anódica (i) versus velocidade de varrimento de potencial (v), para a monocamada formada por $\mathrm{FcCO}\left(\mathrm{CH}_{2}\right)_{5} \mathrm{SH}$ [27]. $R^{2}=0.999$. 
percloratos, pois estes formam um par iónico com a espécie catiónica resultante da oxidação do ferroceno, o ferricínio, estabilizando-a e desviando o potencial formal para valores mais negativos do que, por exemplo, iões sulfato $[5,7,16]$.

\section{COMENTÁRIO FINAL}

Através da breve perspectiva dada, bem como dos resultados apresentados, torna-se evidente que o método "Self-Assembled" é eficaz na construção de monocamadas estáveis e organizadas de alquiltiois em ouro.

O elevado número de estudos de monocamadas SA nas últimas duas décadas é comprovativo da importância destes sistemas em aplicações futuras. Nomeadamente, como já foi referido, na área da biotecnologia e electrónica. Muitos dos artigos mais recentes focam a imobilização de biomoléculas em monocamadas deste tipo para a construção de biossensores.

Verifica-se, no entanto, que são ainda necessários estudos mais fundamentais, tanto a nível estrutural, como no âmbito do mecanismo de transferência electrónica e determinação de parâmetros cinéticos, para uma melhor compreensão do comportamento electroquímico e estabilidade destes sistemas. Neste contexto, a utilização de técnicas estruturais acopladas à electroquímica poderá dar um contributo importante. Contudo, é de referir que a adaptação de tais técnicas a experiências in situ, objecto de nossa investigação actual, onde se faz variar o potencial do eléctrodo modificado, apresenta diversas dificuldades experimentais, que esperamos ultrapassar com sucesso.

A investigação de novos sistemas, pela introdução de diferentes grupos funcionais nas cadeias de hidrocarbonetos e utilização de substratos alternativos, abrirá certamente mais oportunidades de aplicabilidade das monocamadas SA.
1 Poro - Local onde a superfície do eléctrodo está exposta ao electrólito, por não estarem adsorvidas moléculas nem haver bloqueio dos grupos de cauda de moléculas vizinhas.

2 Defeito - Local onde as moléculas e iões do electrólito se podem aproximar da superfície do eléctrodo a uma distância inferior à espessura da monocamada.

\section{REFERÊNCIAS}

1. K. Shimazu, I. Yagi, Y. Sato, K. Ousaki, Langmuir, 8 (1992) 1385-1387.

2. Y. Sato, H. Itoigawa, K. Ousaki, Bulletin of Chemical Society of Japan, 66 (1993) 1032-1037.

3. C. D. Bain, E. B. Troughton, Y. Tao, J. Evall, G.M. Whitesides, R. G. Nuzzo, Journal of American Chemical Society, 111 (1989) 321-325.

4. H. Shen, J. E. Mark, C. J. Seliskar, H. B. Mark, W. R. Heineman, Journal of Solid State Electrochemistry, 1 (1997) 148-154.

5. A. J. Bard, 1. Rubinstein, Electroanalytical Chemistry: A Series of Advances, vol. 19 Marcel Dekker Inc., New York, 1996.

6. R. C. Sabapathy, S. Bhattacharyya, M. C. Leavy, W. E. Clealand, C. L. Hussey, Langmuir, 14 (1998) 124-136.

7. K. Shimazu, I. Yagi, Y. Sato, K. Ousaki, Journal of Electroanalytical Chemistry, 372 (1994) 117-124.

8. G. K. Rowe, S. E. Creager, Langmuir, 10 (1994) 1186-1192.

9. Y. Kim, R. L. McCarley, A. I. Bard, Langmuir, 9 (1993) 1941-1944

10. S. E. Creager, L.A. Hockett, G. K. Rowe, Langmuir, 8 (1992) 854-861.

11. T. Kondo, M. Takechi, Y. Sato, K. Ousaki, Journal of Electroanalytical Chemistry, 381 (1995) 203209.

12. Y. Sato, F. Mizutani, K. Shimazu, S. Ye, K. Ousaki, Journal of Electroanalytical Chemistry, 434 (1997) 115-119.
13. H. C. Long, J.J. Donohue, D. A. Buttry, Langmuir, 7 (1991) 2196-2202.

14. K. Shimazu, Y. Sato, I. Yagi, K. Ousaki, Bulletin of Chemical Society of Japan, 67 (1994) 863-865.

15. Y. Sato, K. Ousaki, Langmuir, 13 (1997) $3157-$ 3161.

16. D. D. Popenoe, R. S. Deinhammer, M. D. Porter, Langmuir, 8 (1992) 2521-2530.

17. M.D. Porter, T. B. Bright, D. L. Allara, C. E. D. Chidsey, Journal of the American Chemical Society, 109 (1987) 3559-3568.

18. R. Greef, Thin Solid Films, 233 (1993) 32-39.

19. T. Ohtshuka, Y. Sato, K. Uosaki, Langmuir, 9 (1994) 3658-3662.

20. J. D. Green, M. T, McDermott, M. D. Porter, Journal of Physical Chemistry, 100 (1996) 1334213345.

21. M. M. Walczak, C. A. Alves, B. D. Lamp, M. D. Porter, Journal of Electroanalytical Chemistry, 438 (1997) 91-97,

22. C. E. D. Chidsey, C. R. Bertozzi, T. M. Putvinski, A. M. Mujsce, Journal of the American Chemical Society, 112 (1990) 4301-4306.

23. K. Weber, L. Hockett, S. Creager, Journal of Physical Chemistry B, 101 (1997) 8286-8291.

24. H. O. Finklea, D. D. Hanshew, Journal of the American Chemical Society, 114 (1992) 31733181.

25. J. F. Smalley, S. W. Weldberg, C. E. D. Chidsey, M. R. Linford, M. D. Newton, Y. Liu, Journal of Physical Chemistry, 99 (1995) 13141-13149.

26. S. E. Creager, G. K. Rowe, Journal of Electroanalytical Chemistry, 420 (1997) 291-299.

27. Trabalho não publicado, desenvolvido no âmbito do Doutoramento de A. S. Viana (1998).

28. Southampton Electrochemistry Group, Instrumental Methods in Electrochemistry, Ellis Horwood, Chichester, UK, 1995. 


\section{๑) SHIMADZU}

Testing Material Division

$>$ Ensaios físicos de materiais:

- Tracção

- Compressão

- Flexão

$>$ Extensometria
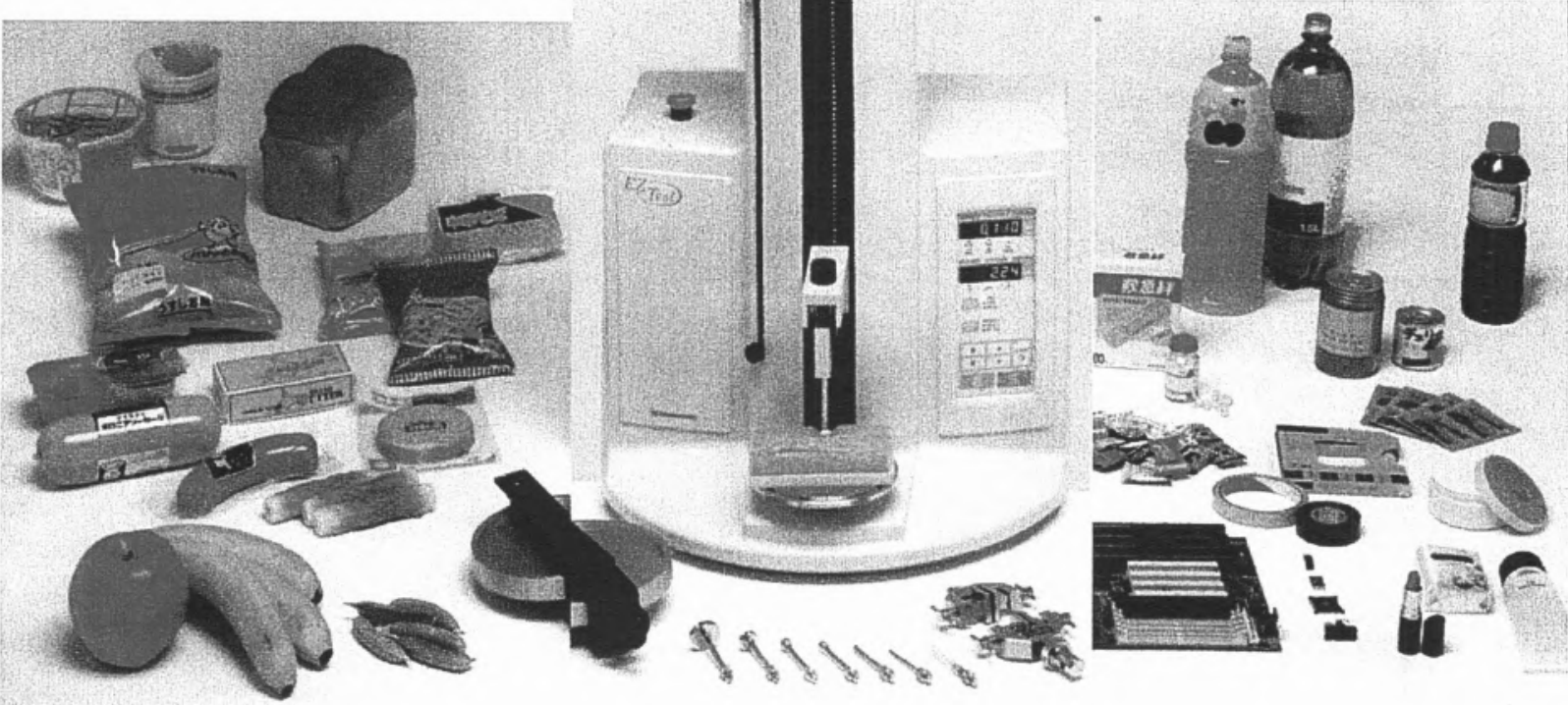

Outros equipamentos que representamos EXCLUSIVAMENTE:

AsD

Fetsche

ตำำ

IKA: $\mathbf{X}$
(Japão) - Balanças de precisão

(Alemanha) - Moinhos, análise granulométrica (peneiros)

(Alemanha) - Estufas (universais, incubação, vazio, baixa temperatura)

(Alemanha) - Equipamento básico de laboratórios, nomeadamente:

Agitadores (mecânicos/magnéticos/hélice), dispersor (UltraTurrax)...

$$
\text { LISBOA PORTO }
$$

